6-1-1997

\title{
Anharmonic Potentials in Supercooled Liquids: The Soft-Potential Model
}

Ulrich Zürcher

Cleveland State University, u.zurcher@csuohio.edu

T. Keyes

Follow this and additional works at: https://engagedscholarship.csuohio.edu/sciphysics_facpub

Part of the Physics Commons

How does access to this work benefit you? Let us know!

Publisher's Statement

(C1997 American Physical Society

\section{Repository Citation}

Zürcher, Ulrich and Keyes, T., "Anharmonic Potentials in Supercooled Liquids: The Soft-Potential Model" (1997). Physics Faculty Publications. 268.

https://engagedscholarship.csuohio.edu/sciphysics_facpub/268

This Article is brought to you for free and open access by the Physics Department at EngagedScholarship@CSU. It has been accepted for inclusion in Physics Faculty Publications by an authorized administrator of

EngagedScholarship@CSU. For more information, please contact library.es@csuohio.edu. 


\title{
Anharmonic potentials in supercooled liquids: The soft-potential model
}

\author{
U. Zürcher* and T. Keyes \\ Department of Chemistry, Boston University, Boston, Massachusetts 02215
}

(Received 23 October 1996)

\begin{abstract}
Instantaneous normal modes (INM) are the harmonic approximation to liquid dynamics. This is an extension of the phonon description of lattice dynamics, in which case Bloch's theorem shows that all modes are extended. Long-range order is destroyed in liquids and glasses, and the INM spectrum has contributions from both extended and localized modes. We use the soft-potential mode to describe localized modes. This model is a high-temperature extension of the standard two-level-system model for glasses. The equilibrium position of any atom in the liquid has only temporary character, and relaxation processes in the liquid are associated with particles hopping over potential energy barriers. Barrier tops have negative curvature so that an INM spectrum has an imaginary frequency (unstable) lobe in addition to the conventional stable mode contributions; conversely the unstable modes carry information about diffusion. We derive analytic expressions for the frequency and temperature dependence of the unstable lobe that are in agreement with results from computer simulations. Self-diffusion of particles in the liquid is governed by the fraction of unstable modes originating from doublewell potentials. For the diffusion constant, we find a crossover behavior from Arrhenius temperature dependence to Zwanzig-Bässler dependence. We find an explicit expression for the distribution of barrier heights. In agreement with Stillinger's inherent structure approach to glass-forming liquids, this distribution is uniform, or Gaussian, for high and low temperatures, respectively. [S1063-651X(97)03706-9]
\end{abstract}

PACS number(s): 47.10.+g, 63.50.+x, 64.70.Pf

\section{INTRODUCTION}

Unlike liquids, solids are capable of elastic resistance to shear stress. It is this property that explains the rigidity of solids and the fluidity of liquids. The rigidity of solids is in agreement with the concept that atoms in a solid oscillate around mechanical equilibrium configurations. Nevertheless, Maxwell suggested that despite the characteristic fluidity of liquids, atoms in a liquid vibrate around equilibrium positions [1]. In order to reconcile this apparent contradiction, Frenkel pointed out that for any atom in a liquid, its equilibrium position is not permanent but rather has temporary character [2]. Each atom performs oscillations about an equilibrium position during a certain time $\tau_{M}$ ("Maxwell time"), until the atom jumps to a new equilibrium position at some distance, which is of the same order of magnitude as the mean distance between adjacent atoms. In this new position, the atom is surrounded, partially at least, by new neighbors.

Zwanzig evokes a similar picture of liquid dynamics in his derivation of the relation between self-diffusion and viscosity of liquids (Stokes-Einstein relation) [3]. Local minima of the potential energy surface of a many-body system divide the configuration space of the system into smaller "cells.", Most of these cells are expected to correspond to amorphous configurations, while others correspond to crystalline (or slightly disordered) configurations. The atoms perform approximate harmonic oscillations around their respective equilibrium positions until the liquid finds a saddle point on the potential energy surface and jumps to another cell. Zwanzig assumes that these harmonic oscillations are described by

\footnotetext{
*Electronic address: zurcher@chem.bu.edu

†Electronic address: keyes@chem.bu.edu $¥$

World wide web: http://chem.bu.edu/ keyes
}

a Debye spectrum, characterized by longitudinal and transverse sound velocities. Jumps between different cells are characterized by a waiting time distribution, approximated by a single exponential function. The diffusion constant then follows from a Green-Kubo formula and obeys a StokesEinstein law. However, as noted already by Zwanzig, this argument does not give an independent estimate for the rate of jumps between cells.

This solidlike approach to liquid dynamics has gained renewed attention in recent years primarily from moleculardynamics simulations $[4,5]$. Expanding the total potential energy around some arbitrary configuration, the second term defines the dynamic matrix. The eigenmodes of the liquid ("instantaneous normal modes," INM) are then defined by diagonalizing the dynamic matrix. Unlike the spectrum of a solid, the INM spectrum consists of both stable $\left(\omega^{2}>0\right)$ and unstable $\left(\omega^{2}<0\right)$ eigenfrequencies. Unstable modes lead to an exponential time dependence and may be identified with barrier crossings between two equilibrium configurations in the liquid. In fact, a relation has been proposed between the fraction of unstable modes $f_{u}=\int_{0}^{\infty} \rho_{u}(\nu) d \nu$ and the selfdiffusion constant, $D \sim T^{1 / 2} f_{u} /\left(1-f_{u}\right)[4,6]$. Here, $\rho_{u}(\nu)$ is the density of unstable modes and $\omega=i \nu$.

Diagonalization of the dynamic matrix yields three zero modes corresponding to rigid translations in three perpendicular directions. The remaining modes give the INM spectrum that shows a linear behavior around $\omega=0, \rho(\omega) \sim|\omega|$ (using the standard convention that the imaginary lobe is plotted along the negative frequency axis). For a system of 500 soft spheres, it was shown that the unstable part of the INM spectrum contains both extended and localized modes [7]. Such localization is a consequence of structural disorder in the system and has previously been found for the same system of soft spheres at zero temperature [8]. For unstable modes, the potential energy profile along eigendirections is 
analyzed in Ref. [7]. It was found that modes with $\nu<\nu_{c}$ correspond to saddle points in single-well potentials, while modes with $\nu>\nu_{c}$ correspond to the system being near the top of double-well barriers.

The fraction of unstable modes increases with increasing temperature. The temperature and frequency dependence of the unstable INM spectrum was analyzed by Keyes for liquids in the supercooled phase [9] and by Vijayadamodar and Nitzan for liquids in the normal phase [10]. In the normal phase, the density follows $\rho_{u}(\nu)=A \nu \exp \left(-B \nu^{2} / T\right)$, while the temperature dependence is stronger in the supercooled phase, $\rho_{u}(\nu)=A \nu \exp \left(-C \nu^{4} / T^{2}\right)$. Here, units are such that $k_{B}=1, A$ is a temperature-dependent prefactor, and $B$ and $C$ are constants. Because unstable modes with $\nu<\nu_{c}$ do not contribute to transport in the liquid [7], the diffusion constant is proportional to the fraction of unstable modes with frequencies $\nu>\nu_{c}$, i.e., $D \propto f_{u}^{\prime}=\int_{\nu_{c}}^{\infty} \rho_{u}(\nu) d \nu$. In turn, this relation suggests an exponential temperature dependence of the viscosity, $\eta \sim 1 / D$. We have Arrhenius temperature dependence in the normal phase, $\ln \eta \sim$ const $/ T$ [11], and ZwanzigBässler temperature dependence in the supercooled phase, $\ln \eta \sim$ const $/ T^{2}$ [12].

The glass temperature is arbitrarily defined as the temperature at which $\log _{10} \eta=13$ (with $[\eta]=$ poise). Angell proposed a classification of glass-forming liquids that is based on the temperature dependence of the viscosity [13]. In strong liquids, Arrhenius behavior is observed from high temperatures where $\log _{10} \eta=-4$ to the glass temperature. In fragile liquids, Arrhenius temperature dependence is observed for low viscosities, $-4 \leqq \log _{10} \eta \lessgtr 2$, while the viscosity varies more strongly than Arrhenius for temperatures close to the glass temperature, $T \gtrsim T_{g}$.

At the glass temperature, an underlying first-order transition is suggested from mode-coupling theories that apply to moderately viscous fluids [14]. In these theories, the friction on a moving particle is ascribed to long-lived density fluctuations in the liquid, which decay by diffusion. The friction increases when the coupling between the particle and the hydrodynamic modes increases, which leads to slower diffusive decay of density fluctuations. This in turn further enhances the coupling, leading to a catastrophe that is identified with the glass transition.

The viscosity is very large at the glass temperature, and the system has properties of a solid on laboratory time scales. Stillinger and Weber have identified rigid aperiodic structures in computer simulations [15]. The motion of individual particles in the liquid is arrested, and their structures are stable to infinitesimal displacements. This requirement is not sufficient, however, since the solid is a thermodynamically stable system. Thus, its structures must be stable to finiteamplitude thermal fluctuations. In fact, Lindemann showed that melting occurs when the width of the mean square thermal displacements of molecules becomes one-tenth of the interparticle spacing [16]. Kirkpatrick and Wolynes have shown that the stability analysis of aperiodic structures and mode-coupling theories are equivalent and lead to the same kind of transition [17].

It was shown in Ref. [15] that groups of atoms create local bistability, and also how they move from one equilibrium position to another along a collective coordinate. This finding gives a microscopic basis for the "two-levelsystem" model (TLS) describing low-temperature thermal and kinetic properties of amorphous solids $[18,19]$. For Lennard-Jones systems, localized modes were identified in computer simulations and their relation to TLS was discussed [20]. A quantitative method was developed for finding TLS's in computer simulations, and a universal theory of low-temperature properties of structural glasses was proposed in Ref. [21]. Glassy anomalies between 1 and $10 \mathrm{~K}$ have been attributed to additional states coexisting with sound waves. In neutron-scattering experiments, these additional modes have been shown to be soft harmonic vibrations with a crossover to anharmonicity at the low frequency end [22]. The soft-potential model describes both the tunneling and the soft vibrational modes in a glass [23], via the addition of two parameters to the standard tunneling model. One parameter is the frequency of the lowest maximum in the vibrational density of the states and is directly accessible to experiment. The second parameter is the product of the effective mass and the square of the atomic displacement, at which the anharmonic part of the potential is dominant. This second parameter thus describes vibrational localization and anharmonicity in the glass. The number of particles participating in a localized mode has been estimated to be $10-100$ [23].

In this paper, we show that the frequency and temperature dependence of the unstable INM spectrum can be derived from the soft-potential model. We relate the distributions of model parameters to the localization of low-lying modes. For each mode, the soft potential defines a barrier height so that the collection of unstable modes implies a barrier height distribution. For high temperatures, we find a uniform distribution, while for low temperatures, the distribution is a Gaussian. Goldstein was first to point out a connection between the temperature dependence of the viscosity and the energy landscape in glass-forming liquids [24]. Properties of the energy landscape are reflected in the susceptibility spectrum of liquids. In the normal and moderately supercooled regime, the spectrum has a single absorption peak. In the supercooled phase, this peak splits into a pair of maxima that correspond to slow $\alpha$ and fast $\beta$ processes [25]. Fast processes have Arrhenius temperature dependence and are operative at the glass temperature. Slow processes have non-Arrhenius temperature dependence and are frozen out at $T_{g}$. We identify the low-temperature Zwanzig-Bässler behavior of the diffusion constant with $\alpha$-relaxation process. The hightemperature Arrhenius behavior is then identified with $\beta$-relaxation processes. We derive an expression for the diffusion constant in terms of the finite mean-square displacement of a particle in the soft potential. It is not surprising that we find such a relation, since the INM spectrum is defined by the short-time expansion of the liquid dynamics [5]. In shorttime experiments, such as neutron and Mössbauer scattering, a (glass) transition has been found in measurements of the mean-square displacement (Debye-Waller factor) [13].

The outline of this paper is as follows. The soft-potential model is introduced in Sec. II, where we focus in particular on the relation between the localization of soft modes and properties of the INM spectrum for $|\omega| \rightarrow 0$. The model is then used in Sec. III to derive the unstable frequency spectrum for low and high temperatures. Parts of this derivation 
have been reported elsewhere [26] but are briefly summarized here for completeness. We find a frequency cutoff that separates contributions to the spectrum from single-well and double-well potentials. We then derive exponential temperature dependence of the diffusion constant in Sec. IV. In Sec. $\mathrm{V}$, we find the distribution of barrier heights in the low- and high-temperature limit. Finally, we summarize and discuss our results in Sec. VI.

\section{THE SOFT-POTENTIAL MODEL}

The Debye model for crystal vibrations fails to explain the low-temperature properties of structural glasses. Below 1 $\mathrm{K}$, the specific heat and the thermal conductivity have a linear and quadratic temperature dependence, respectively, i.e., $C_{p}(T) \propto T$ and $K(T) \propto T^{2}[18,19]$. Within the tunneling model, these universal features of glasses are shown to follow from a distribution of energy differences between the two levels that is smooth on the scale of $k_{B} T$.

At higher temperatures, the thermal conductivity reaches a plateau and the specific heat rises faster than $T^{3}$. Within the TLS model for glasses, the thermal conductivity above the plateau region is explained by phonons scattering off TLS's. This mechanism is critically analyzed in Ref. [27]. Alternatively, Karpov and Parshin suggested a mechanism in which anharmonic modes scatter off the TLS [28]. This model ascribes the linear temperature dependence of thermal conductivity above the plateau temperature to a linear decrease of the scattering rate of these heat-carrying states. In the model of Karpov, Klinger, and Ignatiev [29], and others [30], both the tunneling and soft vibrational states are described by soft anharmonic potentials with locally varying parameters. Buchenau and co-workers find that the two-level system and the low-energy vibrational states can be explained by the same distribution of localized modes [22].

Furthermore, low-frequency $\left(\nu \sim 1-100 \mathrm{~cm}^{-1}\right)$ Raman spectra of glasses have unique features that are attributed to collective excitations involving 10 to 100 atoms [31]. The spectra have anharmonic contributions due to relaxation in double-well potentials and harmonic contributions from motions in single-well potentials (the so-called Boson peak). In Ref. [32], fast picosecond relaxation in supercooled liquids is explained using soft vibrational modes and their damping.

The soft-potential model assumes modes with an effective mass $M$ and a stabilizing fourth-order term [33],

$$
V_{0}(x)=W\left[-D_{2} x^{2}+x^{4}\right] .
$$

Because $x$ is a reduced coordinate, $[x]=1$, the coefficient $D_{2}$ is dimensionless as well. It follows that the square of frequency, $\omega^{2}=d^{2} V / d x^{2}$, has the dimension of an energy, $\left[\omega^{2}\right]=[W]$. The restoring force constant is a random variable characterized by a uniform probability distribution $p_{2}\left(D_{2}\right)$,

$$
p_{2}\left(D_{2}\right)=p_{2}^{0}=\text { const }, \quad 0<D_{2}<\frac{\Omega^{2}}{4 W} \text {. }
$$

Here, we introduce a high-frequency cutoff for soft vibrational modes $\Omega$. At zero temperature, the density of states then follows $g(\omega)=2 \omega p_{2}\left(\omega^{2} / 4 W\right) \sim \omega$. This linear dependence of the density of states is consistent with localized behavior of soft modes [34].

In Ref. [35], the additional specific heat of supercooled liquids is explained by contributions from configurational modes. These configurational modes are identified as local stress and are described by a linear term in the soft potential,

$$
V(x)=W\left[D_{1} x-D_{2} x^{2}+x^{4}\right] .
$$

Because the external field is not included in the definition of the internal energy of the system, the change $\delta V$ follows, $d \delta V=W D_{1} d\langle x\rangle$. Since $d\langle x\rangle=\left(\partial\langle x\rangle / \partial D_{1}\right) d D_{1}$, we have by integration,

$$
\delta V=W \int D_{1} \frac{\partial\langle x\rangle}{\partial D_{1}} d D_{1}
$$

Here, the average is taken with respect to the Boltzmann distribution $\exp [-V(x) / T]$ (in units such that $k_{B}=1$ ),

$$
\frac{\partial\langle x\rangle}{\partial D_{1}}=-\frac{W}{T}\left[\left\langle x^{2}\right\rangle-\langle x\rangle^{2}\right]
$$

We evaluate the right-hand side for zero restoring force constant, $D_{2}=0$, and find $\left\langle x^{2}\right\rangle=0.338(T / W)^{1 / 2}$ and $\langle x\rangle=0$. Equation (2.4) then gives the energy required to generate small stress in the liquid,

$$
\delta V=-0.169 \frac{W^{3 / 2}}{T^{1 / 2}} D_{1}^{2} .
$$

The energy required to generate a small stress $D_{1}$ at $D_{2}=0$ follows $\delta W=-\delta V$. Buchenau and co-workers argue [33] that thermal stress is frozen in the liquid at $T_{g}$ and propose that the Boltzmann factor $\exp \left(-\delta W / T_{g}\right)$ gives the distribution of the linear coefficient $D_{1}$,

$$
p_{1}\left(D_{1}\right)=0.231\left(\frac{T_{g}}{W}\right)^{3 / 4} \exp \left[-0.169\left(\frac{W}{T_{g}}\right)^{3 / 2} D_{1}^{2}\right] .
$$

That is, the coefficient of the linear term of the soft potential is a Gaussian random variable with variance $\left\langle D_{1}^{2}\right\rangle=3.96\left(T_{g} / W\right)^{3 / 2}$. For temperatures $T \gg T_{g}$, the liquid is capable of rearranging its atomic configurations such that thermal stress vanishes. In the limit $T_{g} \rightarrow 0$, thermal stress vanishes at any nonzero temperature,

$$
p_{1}\left(D_{1}\right)=\delta\left(D_{1}\right), \quad T_{g} \rightarrow 0 .
$$

In Eq. (2.7), we neglect configurational rearrangements in the liquid, and assume that relaxation in supercooled liquids is caused by particles hopping between potential energy minima that are frozen in the liquid. Such rearrangements are important at higher temperatures, and give rise to thermal stress that depends explicitly on temperature. Stress fields in glass-forming liquids have been investigated in moleculardynamics simulations [36]. It is found that the stress has long-range order in the supercooled phase, while the normal fluid cannot support this stress field.

The localization of soft modes is discussed in Ref. [37]. The energy to distort a stable structure increases with the 
spatial extent of the instability. This restricts the participating atoms to the close neighborhood of a single atom. A careful analysis gives an estimate of up to 100 atoms participating in a localized mode [23]. This large number of particles may pose a difficulty in distinguishing between localized and extended modes in computer simulations. They rarely involve more than 1000 particles and are thus capable of showing localization of 30 particles. For an extended mode, the atomic displacements are small, so that the anharmonicity is experienced only via the combined action of all other modes.

\section{DENSITY OF UNSTABLE MODES}

In the absence of thermal stress $D_{1}=0$, the soft potential describes symmetric double wells, $V_{0}(x)=W\left[-D_{2} x^{2}+x^{2}\right]$. At zero temperature, the density of states contains only stable modes $g(\omega)=2 \omega p_{2}\left(\omega^{2} / 4 W\right)$ for $0<\omega<\Omega$. For $D_{1}$ $\neq 0$, the soft potential describes both single and double wells, and the density of states depends on the distributions of both $D_{1}$ and $D_{2}$. At nonzero temperatures, the coordinate of each soft mode is weighed by the Boltzmann factor $\exp [-V(x) / T]$. The density of states then follows as

$$
g(\omega)=2 \omega G\left(\omega^{2}\right),
$$

where $G\left(\omega^{2}\right)$ is the density of the square of frequencies,

$$
G\left(\omega^{2}\right)=\left\langle\delta\left(\frac{d^{2} V}{d x^{2}}-\omega^{2}\right)\right\rangle .
$$

Here, the average is taken with respect to the coordinate $x$ and the parameters of the soft potential. At nonzero temperatures, the density of states contains stable $\left(\omega^{2}>0\right)$ as well as unstable modes $\left(\omega^{2}<0\right)$. The fraction of unstable modes increases with increasing temperature.

A temperature scale enters via the variance of the linear term of the soft potential, $\left\langle D_{1}^{2}\right\rangle=3.96\left(T_{g} / W\right)^{3 / 2}$. We consider the supercooled phase of the liquid above the glass temperature, $T>T_{g}$. Introducing scaled coefficients and coordinates,

$$
\begin{aligned}
D_{1} & =\left(\frac{T_{g}}{W}\right)^{3 / 4} \widetilde{D_{1}}, \\
D_{2} & =\left(\frac{T_{g}}{W}\right)^{1 / 2} \widetilde{D_{2}}, \\
x & =\left(\frac{T_{g}}{W}\right)^{1 / 4} \widetilde{x},
\end{aligned}
$$

the ratio $V / T$ follows,

$$
\frac{V}{T}=\frac{T_{g}}{T}\left[\widetilde{D_{1}} \tilde{x}-\widetilde{D}_{2} \widetilde{x}^{2}+\widetilde{x}^{4}\right]
$$

so that the temperature enters the density of states via the combination $T_{g} / T$ only. In particular, the Boltzmann factor is independent of the energy scale $W$. Below we take the limit $W \rightarrow 0$ and then consider the limits $T \rightarrow 0$ and $T_{g} \rightarrow 0$, such that the ratio $T / T_{g}$ is constant. We demonstrate that in this limit, the density of states is uniquely defined.
Furthermore, we may calculate the density of states for a constant temperature $T$ and then study two limiting cases by varying the glass temperature $T_{g}$. For $T / T_{g}=O(1)$, we recover the low-temperature limit while for $T / T_{g} \rightarrow \infty$, the high-temperature limit follows. In the latter case, $T_{g}=0$ and the linear term of the soft potential vanishes, i.e., $p_{1}\left(D_{1}\right)=\delta\left(D_{1}\right)$.

Unstable modes $\omega^{2}<0$ have imaginary frequencies, $\omega=i \nu$. From Eq. (3.2), the density of unstable modes follows:

$$
G_{u}\left(\nu^{2}\right)=\left\langle\delta\left(\frac{d^{2} V}{d x^{2}}+\nu^{2}\right)\right\rangle .
$$

For a fixed pair of coefficients $\left(D_{1}, D_{2}\right)$, we first take the average with respect to the coordinate $x$. Since $d^{2} V / d x^{2}=-\nu^{2}=W\left[-2 D_{2}+12 x^{2}\right]$, the roots are independent of $D_{1}$,

$$
x_{\nu}^{ \pm}= \pm \frac{1}{\sqrt{12}} \sqrt{2 D_{2}-\nu^{2} / W} .
$$

It follows that for a fixed frequency $\nu$, the coefficient $D_{2}$ is bounded from below,

$$
D_{2} \geqslant D_{c}=\frac{\nu^{2}}{2 W} .
$$

Because the coordinate is weighted by the Boltzmann factor $\exp [-V(x) / T]$, the average follows as

$$
\left\langle\delta\left(\frac{d^{2} V}{d x^{2}}+\nu^{2}\right)\right\rangle_{x}=\frac{1}{N_{x}}\left\{\exp \left(-\frac{V\left(x_{\nu}^{+}\right)}{T}\right)+\exp \left(-\frac{V\left(x_{\nu}^{-}\right)}{T}\right)\right\},
$$

where

$$
V\left(x_{\nu}^{ \pm}\right)=W D_{1} x_{\nu}^{ \pm}+V_{0}\left(x_{\nu}^{ \pm}\right) .
$$

In Eq. (3.10), the normalization is given by $N_{x}=\int_{-\infty}^{\infty} \exp [-V(x) / T] d x$. For $D_{1}=0$, the potential has two equivalent minima,

$$
V_{0}\left(x_{\text {min }}^{ \pm}\right)=-\frac{D_{2}^{2}}{4}
$$

at the coordinates

$$
x_{\min }^{ \pm}= \pm \sqrt{\frac{D_{2}}{2}} .
$$

The double-well structure is preserved for small values of $D_{1}$. Defining $Q=-\left(D_{2} / 6\right)^{3}+\left(D_{1} / 8\right)^{2}$, double and single wells follow for $Q<0$ and $Q>0$, respectively. For a doublewell potential, we thus have the inequality $D_{2}^{3}>(27 / 8) D_{1}^{2}$. We recall that $D_{1}$ is a Gaussian random variable with zero mean and variance $\left\langle D_{1}^{2}\right\rangle=3.96\left(T_{g} / W\right)^{3 / 2}$. Thus, the soft potential has double-well structure for sufficiently large restoring force constants, $D_{2}^{3}>13.36\left(T_{g} / W\right)^{3 / 2}$. For a fixed frequency $\nu$, the coefficient $D_{2}$ is bounded from below $D_{2}>\nu^{2} / 2 W$; cf. Eq. (3.9). We conclude that for large fre- 
quencies, $\nu>\nu_{c}$, the density of unstable states is dominated by contributions from double wells, while for small frequencies, $\nu<\nu_{c}$, it is dominated by contributions from single wells. This frequency cutoff $\nu_{c}$ depends on the product of the glass temperature $T_{g}$ and the energy scale $W$,

$$
\nu_{c} \simeq 2.18\left(T_{g} W\right)^{1 / 4} .
$$

In the limit $T_{g} \rightarrow 0$, the cutoff is arbitrarily small, $\nu_{c} \rightarrow 0$, and all unstable modes originate from double-well potentials.
For a large restoring force constant, the linear term of the soft potential is a small perturbation. In quadratic order in $D_{1}$, the coordinates of the potential minima are given by $\widetilde{x}_{\min }^{ \pm}= \pm \sqrt{D_{2} / 2}-D_{1} / 4 D_{2} \mp(3 / 16) \sqrt{D_{2} / 2} D_{1}^{2} / D_{2}^{3}$. The potential minima then follow as $\widetilde{V}_{\min }=W\left[-D_{2}^{2} / 4\right.$ $\left.\pm D_{1} \sqrt{D_{2} / 2}-D_{1}^{2} / 8 D_{2}\right]$. For a double-well potential, we have $D_{2}^{2} / 4 \gg D_{1}^{2} / 8 D_{2}$, and the quadratic correction of the potential can be neglected. It follows that the Boltzmann distribution is a superposition of two Gaussians, and the normalization is given by

$$
N_{x}=\sqrt{\frac{\pi T}{2 W D_{2}}} \exp \left(-\frac{V_{0}\left(x_{\min }^{ \pm}\right)}{T}\right)\left\{\exp \left(-\frac{W D_{1}}{T} x_{\min }^{+}\right)+\exp \left(-\frac{W D_{1}}{T} x_{\min }^{-}\right)\right\},
$$

where $V_{0}\left(x_{\min }^{+}\right)=V_{0}\left(x_{\min }^{-}\right) \equiv V_{0}\left(x_{\min }^{ \pm}\right)$.

We choose as a reference point the inflection point of the symmetric double well $d^{2} V_{0}(x) / d x^{2}=0$,

$$
x_{0}=\sqrt{\frac{D_{2}}{6}}
$$

and find for nonzero frequencies $\nu^{2} \neq 0$,

$$
\begin{aligned}
\left\langle\delta\left(\frac{d^{2} V}{d x^{2}}+\nu^{2}\right)\right\rangle_{x} & \sqrt{\frac{2 W D_{2}}{\pi T}} \exp \left(-\frac{1}{T}\left[V_{0}\left(x_{0}\right)-V_{0}\left(x_{\min }\right)\right]-\frac{W\left|D_{1}\right|}{T}\left[x_{\min }-x_{0}\right]\right) \\
& \times \exp \left(-\frac{1}{T}\left[V_{0}\left(x_{\nu}\right)-V_{0}\left(x_{0}\right)\right]-\frac{W\left|D_{1}\right|}{T}\left[x_{0}-x_{\nu}\right]\right),
\end{aligned}
$$

where $x_{\nu}=x_{\nu}^{+}=-x_{\nu}^{-}$and $x_{\min }=x_{\min }^{+}=x_{\min }^{-}$. The density of states of unstable modes now follows by taking the limit $W \rightarrow 0$ and then considering the average with respect to the parameters of the soft potential; cf. the discussion following Eq. (3.6).

Because the potential $V_{0}(x)$ vanishes as $W \rightarrow 0$, the argument of the exponential function on the RHS is small in this limit. We first take the average with respect to the uniform distribution of $D_{2}$ and truncate the cumulant expansion at the first term,

$$
\begin{aligned}
\left\langle\delta\left(\frac{d^{2} V}{d x^{2}}+\nu^{2}\right)\right\rangle_{x, D_{2}} \simeq & \sqrt{\frac{2 W\left\langle D_{2}\right\rangle}{\pi T}} \exp \left(-\frac{1}{T}\left\langle\left[V_{0}\left(x_{0}\right)-V_{0}\left(x_{\min }\right)\right]\right\rangle_{D_{2}}-\frac{W\left|D_{1}\right|}{T}\left\langle\left[x_{\min }-x_{0}\right]^{2}\right\rangle_{D_{2}}^{1 / 2}\right) \\
& \times \exp \left(-\frac{1}{T}\left\langle\left[V_{0}\left(x_{\nu}\right)-V_{0}\left(x_{0}\right)\right]\right\rangle_{D_{2}}-\frac{W\left|D_{1}\right|}{T}\left\langle\left[x_{0}-x_{\nu}\right]^{2}\right\rangle_{D_{2}}^{1 / 2}\right) .
\end{aligned}
$$

Here, we replaced $\left\langle\left[x_{\min }-x_{0}\right]\right\rangle_{D_{2}}$ and $\left\langle\left[x_{0}-x_{\nu}\right]\right\rangle_{D_{2}}$ by $\left\langle\left[x_{\min }-x_{0}\right]^{2}\right\rangle_{D_{2}}^{1 / 2}$ and $\left\langle\left[x_{0}-x_{\nu}\right]^{2}\right\rangle_{D_{2}}^{1 / 2}$, respectively. We recall from Eq. (3.9) that for a fixed frequency $\nu$, the restoring constant is bounded from below, $D_{2} \geqslant \nu^{2} / 2 W$. We readily find $\left\langle D_{2}\right\rangle \simeq\left[\Omega^{2} / 8 W+\nu^{2} / 4 W\right]$. Equations (3.8) and (3.16) then give $\left\langle x_{0}^{2}+x_{\nu}^{2}\right\rangle=\Omega^{2} / 24 W$ and $\left\langle x_{0} x_{\nu}\right\rangle=(12 W)^{-1} \sqrt{\Omega^{4} / 16-\nu^{4} / 4}$ $\simeq \Omega^{2} / 48 W-\nu^{4} / 24 W \Omega^{2}$ for $\nu^{2} \rightarrow 0$. It follows that

$$
\left\langle\delta\left(\frac{d^{2} V}{d x^{2}}+\nu^{2}\right)\right\rangle_{x, D_{2}} \simeq \sqrt{\frac{\Omega^{2}}{4 \pi T}} \exp \left(-\frac{1}{432} \frac{\Omega^{4}}{W T}-\frac{1}{3 \sqrt{10}} \frac{W^{1 / 2} \Omega\left|D_{1}\right|}{T}-\frac{1}{144} \frac{\Omega^{2} \nu^{2}}{W T}-\frac{1}{\sqrt{12}} \frac{W^{1 / 2} \nu^{2}\left|D_{1}\right|}{\Omega T}\right) .
$$

The quantity $G_{u}(0)$ now follows by setting $\nu=0$ and taking the average with respect to $D_{1}$. We have

$$
\left\langle\delta\left(\frac{d^{2} V}{d x^{2}}\right)\right\rangle_{x, D_{2}} \simeq \sqrt{\frac{\Omega^{2}}{4 \pi T}} \exp \left(-\frac{1}{432} \frac{\Omega^{4}}{W T}-\frac{1}{3 \sqrt{10}} \frac{W^{1 / 2} \Omega\left|D_{1}\right|}{T}\right) .
$$


Since $\left\langle D_{1}^{2}\right\rangle=3.96\left(T_{g} / W\right)^{3 / 2}$, the variance of $D_{1}$ diverges as $W \rightarrow 0$. For a fixed energy scale $W$, the combination $W^{1 / 2} \Omega\left|D_{1}\right| / 3 \sqrt{10} T$ becomes large in the limit $\left|D_{1}\right| \rightarrow \infty$. Similarly, the average $\left\langle\exp \left(W^{1 / 2} \Omega\left|D_{1}\right| / 3 \sqrt{10} T\right)\right\rangle_{D_{1}}$ is determined by the behavior of the probability density $p_{1}\left(D_{1}\right)$ for large values of $D_{1}$. For $W>0$, we replace $\left\langle\exp \left(-W^{1 / 2} \Omega\left|D_{1}\right| / 3 \sqrt{10} T\right)\right\rangle_{D_{1}}$ by $1 /\left\langle\exp \left(W^{1 / 2} \Omega\left|D_{1}\right| / 3 \sqrt{10} T\right)\right\rangle_{D_{1}}$ and then consider the limit $W \rightarrow 0$ such that the variance of the scaled Gaussian random variable $D_{1}^{\prime}=W^{3 / 4} D_{1}$ is finite, $\left\langle D_{1}^{\prime 2}\right\rangle<\infty$. A Gaussian integration gives $\left\langle\exp \left(W^{1 / 2} \Omega\left|D_{1}\right| / 3 \sqrt{10} T\right)\right\rangle_{D_{1}}=\exp \left(T_{g}^{3 / 2} \Omega^{2}\right.$ / $\left.20 W^{1 / 2} T^{2}\right)$. We then have

$$
G_{u}(0) \simeq \sqrt{\frac{\Omega^{2}}{4 \pi T}} \exp \left(-\frac{1}{432} \frac{\Omega^{4}}{W T}-\frac{1}{20} \frac{T_{g}^{3 / 2} \Omega^{2}}{W^{1 / 2} T^{2}}\right) .
$$

We introduce a dimensionless frequency and temperature,

$$
\begin{gathered}
\Omega^{\prime}=\frac{\Omega}{\sqrt{W}}, \\
T^{\prime}=\frac{T}{W},
\end{gathered}
$$

and find

$$
G_{u}(0) \simeq \sqrt{\frac{\Omega^{2}}{4 \pi T}} \exp \left(-\frac{1}{432} \frac{\Omega^{4}}{T}-\frac{1}{20} \frac{T_{g}^{3 / 2} \Omega^{2}}{T^{2}}\right),
$$

where we replaced primed quantities by unprimed ones to simplify the notation.

We estimate the relative magnitude of the two terms in the argument of the exponential function by setting $T=T_{g}$. We have $G_{u}\left(0, T=T_{g}\right) \simeq \sqrt{\Omega^{2} / 4 \pi T_{g}} \exp \left(-\Omega^{4} / 432 T_{g}\right.$ $\left.-\Omega^{2} / 20 T_{g}^{1 / 2}\right)$. The first term dominates for $\Omega>4.64 T_{g}^{1 / 4^{g}}$. Since $\nu_{c}=2.18 T_{g}^{1 / 4}$ [cf. Eq. (3.14)], we have for all temperatures $T>T_{g}$,

$$
G_{u}(0) \simeq \sqrt{\frac{\Omega^{2}}{4 \pi T}} \exp \left(-\frac{1}{432} \frac{\Omega^{4}}{T}\right), \quad \nu_{c}<\frac{\Omega}{2} .
$$

That is, $G_{u}(0)$ is independent of the glass temperature $T_{g}$.

We calculate $G_{u}\left(\nu^{2}\right)$ in a similar fashion. We insert Eq. (3.25) into Eq. (3.19),

$$
\frac{G_{u}\left(\nu^{2}\right)}{G_{u}(0)} \simeq \frac{\exp \left(-\Omega^{2} \nu^{2} / 144 W T\right)}{\left\langle\exp \left(W^{1 / 2} \nu^{2}\left|D_{1}\right| / \sqrt{12} \Omega T\right)\right\rangle_{D_{1}}} .
$$

A Gaussian integration gives

$$
\frac{G_{u}\left(\nu^{2}\right)}{G_{u}(0)} \simeq \exp \left(-\frac{1}{144} \frac{\Omega^{2} \nu^{2}}{W T}-\frac{1}{8} \frac{T_{g}^{3 / 2} \nu^{4}}{W \Omega^{2} T^{2}}\right) .
$$

We introduce a dimensionless frequency,

$$
\nu^{\prime}=\frac{\nu}{\sqrt{W}},
$$

cf. Eq. (3.22), and find

$$
\frac{G_{u}\left(\nu^{2}\right)}{G_{u}(0)} \simeq \exp \left(-\frac{1}{144} \frac{\Omega^{2} \nu^{2}}{T}-\frac{1}{8} \frac{T_{g}^{3 / 2} \nu^{4}}{\Omega^{2} T^{2}}\right) .
$$

We observe that the temperature-dependent density of unstable modes is characterized by the glass temperature $T_{g}$ and the upper frequency cutoff $\Omega$.

The density of states is given by $g_{u}(\nu)=2 \nu G_{u}\left(\nu^{2}\right)$; cf. Eq. (3.1). The high-temperature limit is obtained by setting $T_{g}=0$; cf. the discussion following Eq. (3.6). We recover the Arrhenius temperature dependence of the unstable density for liquids in the normal phase that has been proposed by Vijayadamodar and Nitzan [10],

$$
g_{u}(\nu) \simeq 2 G_{u}(0) \nu \exp \left(-\frac{1}{144} \frac{\Omega^{2} \nu^{2}}{T}\right), T \gg T_{g} .
$$

For lower temperatures, $T \gtrsim T_{g}$, we recover the ZwanzigBässler temperature dependence of the unstable density for liquids in the supercooled phase that has first been proposed by Keyes [9],

$$
g_{u}(\nu) \simeq 2 G_{u}(0) \nu \exp \left(-\frac{1}{8} \frac{T_{g}^{3 / 2} \nu^{4}}{\Omega^{2} T^{2}}\right), \quad T \gtrsim T_{g} .
$$

Equations (3.30) and (3.31) together with the frequency cutoff $\nu_{c}=2.18 T_{g}^{1 / 4}$ [cf. Eq. (3.14)], are the central results of this section. Below we discuss implications of the frequency and temperature dependence of the unstable INM spectrum to dynamic properties of the liquid.

\section{DIFFUSION CONSTANT}

Instantaneous normal modes are defined via the shorttime expansion of the equations of motion of individual particles in liquids, and solidlike aspects of liquid dynamics are emphasized. Nevertheless, Keyes and co-workers have demonstrated the usefulness of INM analysis to describe longtime dynamical properties of cold liquids [4]. More recently, collective modes were used in Ref. [38] to describe selfdiffusion in hot liquids above the melting point. The selfdiffusion coefficient is approximated by the ratio of the time spent in a valley $\tau_{v}$ to the time spent in crossing a barrier $\tau_{b}, D \simeq T^{1 / 2} \tau_{b} / \tau_{v}$. For liquid dynamics on short time scales, unstable modes are the signature of barrier crossings. The fraction of unstable modes is given by $f_{u}=\int g_{u}(\nu) d \nu$ [6]. Using transition rate theory, Keyes estimates that short-time and long-time properties are related to each other, $\tau_{b} / \tau_{v}$ $\propto f_{u} /\left(1-f_{u}\right)$ [9]. Several other approximations suggest that any strong $T$ dependence of $D$ follows from the relation, $D(T) \sim f_{u}(T)$.

Unstable modes originate from both double and single wells. Because particle transport follows from hopping processes, unstable modes from single wells should not contribute to the self-diffusion constant. For frequencies $\nu>\nu_{c}$, all unstable modes originate from double wells, whereas for $\nu<\nu_{c}$, as we just have seen, the density of unstable modes has large contributions from single wells. It follows that INM theories of the diffusion constant should employ modes 
with $\nu>\nu_{c}$ only; where $f_{u}$ appeared in earlier work, it should be replaced by $f_{u}^{\prime}=\int_{\nu_{c}}^{\infty} g(\nu) d \nu$. Because the density of states of unstable modes has exponential frequency dependence, a nonzero cutoff gives a fraction that is exponentially small, $f_{u}^{\prime} \sim g_{u}\left(\nu_{c}\right)<1$. It then follows that the diffusion constant is exponentially small as well,

$$
D \sim g\left(\nu_{c}\right) .
$$

In dimensionless units, the cutoff frequency depends only on the glass temperature; cf. Eq. (3.14),

$$
\nu_{c}=2.18 T_{g}^{1 / 4} .
$$

Inserting Eqs. (4.1) and (4.2) into Eq. (3.30), we find Arrhenius behavior [11] for high temperatures,

$$
D \sim \exp \left(-\frac{1}{36} \frac{\Omega^{2} T_{g}^{1 / 2}}{T}\right), T \gg T_{g} .
$$

For lower temperatures, Zwanzig-Bässler behavior [12] follows from Eq. (3.31),

$$
D \sim \exp \left(-2 \frac{T_{g}^{5 / 2}}{\Omega^{2} T^{2}}\right), \quad T \gtrsim T_{g} .
$$

Equations (4.3) and (4.4) give the crossover temperature $T_{c}, \Omega^{2} T_{g}^{1 / 2} / 36 T_{c}=2 T_{g}^{5 / 2} / \Omega^{2} T_{c}^{2}$, or

$$
T_{c}=72 \frac{T_{g}^{2}}{\Omega^{4}} .
$$

At the crossover temperature, the diffusion constant $D_{c}=D\left(T_{c}\right)$ is given by $D_{c} \sim \exp \left(-\Omega^{6} / 2592 T_{g}^{3 / 2}\right)$.

We readily express the crossover behavior of the diffusion constant in terms of characteristic energy scales of the soft potential. We have found non-Arrhenius temperature dependence in the case when the linear term of the soft potential is large compared to the quadratic term. Setting $W D_{1} x=W D_{2} x^{2}$, we find that the linear term is larger than the quadratic term for small displacements $x<x_{c}$, while the quadratic term is larger for $x>x_{c}$. Here, $x_{c}=D_{1} / D_{2}$ and the crossover energy follows as $E_{c}=W D_{1}^{2} / D_{2}$. We replace $D_{1}^{2}$ by its average $\left\langle D_{1}^{2}\right\rangle$ and set the restoring force equal to its maximum value; cf. Eqs. (2.2). This gives a lower bound for energies characterizing thermal stress in the liquid,

$$
E_{\alpha}=16 \frac{T_{g}^{3 / 2}}{\Omega^{2}} .
$$

In the absence of thermal stress, $D_{1}=0$, the soft potential is given by $V_{0}(x)=W\left[-D_{2} x^{2}+x^{4}\right]$. The barrier height of the symmetric double well follows as $\Delta V_{0}=W D_{2}^{2} / 4$. Setting $D_{2}=\Omega^{2} / 4 \mathrm{~W}$, we find the characteristic energy associated with soft vibrational modes in the liquid,

$$
E_{\beta}=\frac{\Omega^{4}}{64} .
$$

In Eqs. (4.6) and (4.7), we introduce dimensionless energies, $E / W \rightarrow E$; cf. Eq. (3.23).
From Eq. (4.5), we have $T_{c} / T_{g}=72 T_{g} / \Omega^{4}$. Since $\left(E_{\alpha} / E_{\beta}\right)^{2 / 3}=102 T_{g} / \Omega^{4}$, we find

$$
\frac{T_{c}}{T_{g}} \simeq 0.7\left(\frac{E_{\alpha}}{E_{\beta}}\right)^{2 / 3} .
$$

At the crossover temperature, the diffusion constant follows,

$$
D\left(T_{c}\right) \sim \exp \left(-0.4 \frac{E_{\beta}}{E_{\alpha}}\right) .
$$

Thus, the crossover between Arrhenius and Zwanzig-Bässler behavior depends only on the ratio $E_{\alpha} / E_{\beta}$. For $E_{\alpha}>E_{\beta}$, we have $T_{c}>T_{g}$ and Zwanzig-Bässler temperature dependence follows for some nonzero temperature range above $T_{g}$. For $E_{\beta}>E_{\alpha}$, on the other hand, the diffusion constant is arbitrarily small at $T=T_{c}$, and Arrhenius temperature dependence follows for all temperatures above $T_{g}$. That is, $E_{\alpha}>E_{\beta}$ applies to fragile liquids, whereas $E_{\beta}>E_{\alpha}$ applies to strong liquids [13].

Stillinger relates various static and dynamic properties of glass-forming liquids to the multidimensional complex topography of the collective potential energy function [39]. The topography of strong liquids is uniformly rough, and only $\beta$-relaxation processes are relevant. In fragile liquids, individual local minima ("basins") are organized in deeper potential energy wells ("craters"). It is only at high temperatures that particles explore regions of the configuration space with uniformly rough topography, while at lower temperatures, particles surmount larger and wider potential energy barriers. This transition gives rise to the bifurcation of the temperature dependence of the peak relaxation frequency in liquids. In the equilibrium liquid range, the single absorption maximum peak has Arrhenius temperature dependence. This single maximum splits into a pair of maxima in the supercooled regime. The peak corresponding to fast $\beta$ ("secondary") relaxation has Arrhenius behavior and persists even in the glassy phase. The peak originating from slow $\alpha$ ("primary") relaxation has non-Arrhenius behavior and is frozen out at the glass temperature. The connection with the present work is made by identifying $E_{\alpha}$ and $E_{\beta}$ with a lower limit of energy barriers of craters and the characteristic energy of basins, respectively.

In fact, a more explicit connection can be made between high-temperature behavior and fast dynamic processes in liquids. In the high-temperature limit, the soft potential describes symmetric double wells. On short time scales, the particles perform harmonic oscillations around the potential minima. The mean square displacement (MSD) of a harmonic oscillator is given by $\left\langle x^{2}\right\rangle_{\omega}=T / \omega^{2}$. Replacing the square of the frequency by the upper cutoff for the restoring force constant, we find the MSD characterizing fast processes in liquids,

$$
\left\langle x^{2}\right\rangle_{\beta}=\frac{4 T}{\Omega^{2}} .
$$

The linear term of the soft potential describes stress that is frozen in the liquid at the glass temperature. For zero restoring force constant, $D_{2}=0$, the MSD is given by 
$\left\langle x^{2}\right\rangle=0.338 T^{1 / 2}$; cf. the discussion following Eq. (2.5). Setting $T=T_{g}$, we find the static contribution to the MSD,

$$
\left\langle x^{2}\right\rangle_{\text {static }}=0.338 T_{g}^{1 / 2} .
$$

We use Eqs. (4.10) and (4.11) to rewrite the hightemperature limit of the diffusion constant,

$$
D \sim \exp \left(-\frac{1}{3} \frac{\left\langle x^{2}\right\rangle_{\text {static }}}{\left\langle x^{2}\right\rangle_{\beta}}\right), \quad T \gg T_{g} .
$$

Buchenau and Zorn report neutron time-of-flight measurements of atomic displacements for frequencies above $10^{10} \mathrm{~Hz}$ in glassy, liquid, and crystalline selenium [40]. They observe a weak temperature dependence of the mean square displacement in the ordered phase and a strong temperaturedependent enhancement in the disordered phase. They find a linear relation between the logarithm of the viscosity and the inverse of the enhancement of the MSD. Since the viscosity is proportional to the inverse of the diffusion constant, this relation is in agreement with our prediction; cf. Eq. (4.12).

\section{DISTRIBUTION OF BARRIER HEIGHTS}

Following our discussion of the diffusion constant, the density of states of unstable modes reflects the topography of the potential energy landscape of liquids. The landscape consists of local minima separated by potential barriers, and is thus characterized by the distribution of barrier heights. In the soft-potential model, we define a barrier height for each pair of parameters $\left(D_{1}, D_{2}\right)$. We have

$$
\Delta V=V\left(x_{\nu}\right)-V\left(x_{\min }\right),
$$

where $x_{\nu}$ and $x_{\min }$ are given by the roots $d^{2} V / d x^{2}+\nu^{2}=0$ and $d V / d x=0$, respectively; cf. Eqs. (3.8) and (3.13). The distribution of barrier heights is then defined as

$$
\mathcal{P}(E ; \nu)=\langle\delta(E-\Delta V)\rangle_{D_{1}, D_{2}} .
$$

Here, $\mathcal{P}(E ; \nu)$ depends on the frequency $\nu$ through the dependence on the coordinate $x_{\nu}$. We calculate the average on the right-hand side by inserting the Fourier representation of the $\delta$ function, $\delta(x)=(1 / 2 \pi) \int_{-\infty}^{\infty} d q \exp (i q x)$. We interchange the order of taking the average with respect to the parameters of the soft potential and integration,

$$
\mathcal{P}(E ; \nu)=\frac{1}{2 \pi} \int_{-\infty}^{\infty} d q \exp (i q E)\langle\exp (-i q \Delta V)\rangle_{D_{1}, D_{2}} .
$$

The discussion of the preceding section suggests that barrier heights have different distributions in the high- and lowtemperature limits. For $T \gg T_{g}$, we can neglect the linear term of the soft potential, $V(x) \simeq V_{0}(x)=W\left[-D_{2} x^{2}+x^{4}\right]$,
$\mathcal{P}(E ; \nu)=\frac{1}{2 \pi} \int_{-\infty}^{\infty} d q \exp (i q E)\left\langle\exp \left(-i q \Delta V_{0}\right)\right\rangle_{D_{2}}, \quad T \gg T_{g}$

For $\quad W \rightarrow 0$, we replace $\left\langle\exp \left(-i q \Delta V_{0}\right)\right\rangle_{D_{2}} \quad$ by $\exp \left(-i q\left\langle\Delta V_{0}\right\rangle_{D_{2}}\right)$ [cf. Eq. (3.18)],

$$
\mathcal{P}(E ; \nu)=\frac{1}{2 \pi} \int_{-\infty}^{\infty} d q \exp \left(i q\left[E-\left\langle\Delta V_{0}\right\rangle_{D_{2}}\right]\right), \quad T \gg T_{g} .
$$

Since

$$
\begin{aligned}
V_{0}\left(x_{\nu}\right)-V_{0}\left(x_{\min }\right)= & {\left[V_{0}\left(x_{\nu}\right)-V_{0}\left(x_{0}\right)\right] } \\
& +\left[V_{0}\left(x_{0}\right)-V_{0}\left(x_{\min }\right)\right]
\end{aligned}
$$

(where $x_{0}$ is the inflection point of $V_{0}(x)$ [cf. Eq. (3.16)]), we readily find $\left\langle\Delta V_{0}\right\rangle_{D_{2}}=\Omega^{2} \nu^{2} / 144 W+\Omega^{4} / 432 W$. We introduce a dimensionless energy

$$
E^{\prime}=\frac{E}{W},
$$

along with the dimensionless frequencies $\Omega^{\prime}=\Omega / \sqrt{W}$ and $\nu^{\prime}=\nu / \sqrt{W}$; cf. Eqs. (3.22) and (3.28). We find $\mathcal{P}(E ; \nu)=(1 / 2 \pi) \int_{-\infty}^{\infty} d q \exp \left(-i q\left[E-\Omega^{2} \nu^{2} / 144-\Omega^{4} / 432\right]\right)$, where we replaced primed quantities by unprimed ones. The $q$ integration now yields $\mathcal{P}(E ; \nu)=\delta\left(E-\nu^{2} \Omega^{2} / 144\right.$ $\left.-\Omega^{4} / 432\right)$. The characteristic energy for fast processes in the liquid is given by $E_{\beta}=\Omega^{4} / 64$; cf. Eq. (4.7). We finally find the barrier height distribution in the high-temperature limit,

$$
\mathcal{P}(E ; \nu)=\delta\left(E-\frac{4}{27} E_{\beta}-\frac{1}{18} \sqrt{E_{\beta}} \nu^{2}\right), \quad T \gg T_{g} .
$$

Thus, fast processes in the liquid are associated with a potential energy landscape that is uniformly rough.

For lower temperatures, the leading frequency dependence of the potential difference $\Delta V$ originates from the linear term of the soft potential,

$$
\Delta V \simeq\left[V_{0}\left(x_{0}\right)-V_{0}\left(x_{\min }\right)\right]-W\left|D_{1}\right|\left[x_{0}-x_{\nu}\right], \quad T \gtrsim T_{g} .
$$

The distribution of barrier heights now follows by taking the average with respect to both parameters of the soft potential [cf. Eq. (5.3)],

$$
\begin{aligned}
\mathcal{P}(E ; \nu)= & (1 / 2 \pi) \int_{-\infty}^{\infty} d q \\
& \times \exp (i q E)\left\langle\operatorname { e x p } \left(-i q\left[V_{0}\left(x_{0}\right)-V_{0}\left(x_{\min }\right)\right]\right.\right. \\
& \left.\left.+i q W\left|D_{1}\right|\left[x_{0}-x_{\nu}\right]\right)\right\rangle_{D_{1}, D_{2}} .
\end{aligned}
$$

For $W \rightarrow 0$, we proceed by first taking the average with respect to the restoring force constant $D_{2}$,

$$
\mathcal{P}(E ; \nu)=\frac{1}{2 \pi} \int_{-\infty}^{\infty} d q \exp \left(i q\left\{E-\left\langle\left[V_{0}\left(x_{0}\right)-V_{0}\left(x_{\text {min }}\right)\right]\right\rangle_{D_{2}}\right\}\right)\left\langle\exp \left(i q W\left|D_{1}\right|\left\langle\left[x_{0}-x_{\nu}\right]^{2}\right\rangle_{D_{2}}^{1 / 2}\right)\right\rangle_{D_{1}}, \quad T \geqslant T_{g}
$$

Using $\left\langle\left[x_{0}-x_{\nu}\right]^{2}\right\rangle_{D_{2}}=\nu^{4} / 12 W \Omega^{2}$, we find 


$$
\mathcal{P}(E ; \nu)=\frac{1}{2 \pi} \int_{-\infty}^{\infty} d q \exp \left(i q\left[E-\Omega^{4} / 432 W\right]\right)\left\langle\exp \left(i q W^{1 / 2} \nu^{2}\left|D_{1}\right| / \sqrt{12} \Omega\right)\right\rangle_{D_{1}}, \quad T \geqslant T_{g}
$$

The parameter $D_{1}$ is a Gaussian random variable with variance $\left\langle D_{1}^{2}\right\rangle=3.96\left(T_{g} / W\right)^{3 / 2}$. In particular, the variance diverges as $W \rightarrow 0$, and we cannot use a cumulant expansion to calculate the average with respect to $D_{1}$; cf. the discussion preceding Eq. (3.21). Rather, a Gaussian integration gives $\left\langle\exp \left(-i q W^{1 / 2} \nu^{2}\left|D_{1}\right| / \sqrt{12} \Omega\right)\right\rangle_{D_{1}}=\exp \left(-q^{2} T_{g}^{3 / 2} \nu^{4} / 8 W^{1 / 2} \Omega^{2}\right)$. We then have

$$
\mathcal{P}(E ; \nu)=\frac{1}{2 \pi} \int_{-\infty}^{\infty} d q \exp \left(i q\left[E-\Omega^{4} / 432 W\right]\right) \exp \left(-\frac{T_{g}^{3 / 2} \nu^{4}}{8 W^{1 / 2} \Omega^{2}} q^{2}\right), \quad T \geq T_{g} .
$$

It follows that $\mathcal{P}(E ; \nu)=\left(\sqrt{2} W^{1 / 4} \Omega / \sqrt{\pi} T_{g}^{3 / 4} \nu^{2}\right) \exp \left(-2 W^{1 / 2} \Omega^{2}\left[E-\Omega^{4}\right]^{2} / T_{g}^{3 / 2} \nu^{4}\right)$.

As above, we introduce a dimensionless energy $E^{\prime}=E / W$ and frequencies $\nu^{\prime}=\nu / \sqrt{W}$ and $\Omega^{\prime}=\Omega / \sqrt{W}$. In Eq. (4.6), we have found the characteristic energy for slow processes in the liquid, $E_{\alpha}=16 T_{g}^{3 / 2} / \Omega^{2}$. We obtain

$$
\mathcal{P}(E ; \nu)=\frac{1}{\sqrt{\pi E_{\alpha} \nu^{4} / 32}} \exp \left(-\frac{32}{E_{\alpha} \nu^{4}}\left[E-\frac{4}{27} E_{\beta}\right]^{2}\right), T \gtrsim T_{g} .
$$

Thus, slow processes in the liquid are associated with a rugged potential energy landscape characterized by a Gaussian distribution of barrier heights.

The two limiting barrier height distributions are in agreement with the temperature dependence of the diffusion constant. High-temperature Arrhenius behavior implies a single potential energy barrier for viscous flow in the liquid, while low-temperature Zwanzig-Bässler behavior of diffusion is characteristic for random energy models [41].

\section{SUMMARY AND DISCUSSION}

The solidlike approach to liquid dynamics has been revived in recent years largely by computer simulations. Instantaneous normal modes are obtained by diagonalizing the dynamic matrix for a representative configuration that has been selected from a molecular-dynamics simulation. The INM spectrum is obtained from a snapshot of the liquid and describes the dynamics at short times. Nevertheless, following Zwanzig's normal mode description of self-diffusion in liquids, the INM spectrum has been used to describe liquid dynamics on long time scales as well. This paper is a step towards a clearer understanding of the relation between properties of the INM spectrum and those governing the time evolution of the liquid on long time scales. In supercooled liquids, the viscosity varies over more than 10 decades. Goldstein, and later Stillinger, showed that exponential temperature dependence of viscous flow can be understood from a topographic viewpoint of the potential energy landscape in liquids. In this paper, we have shown that exponential frequency and temperature dependence of the unstable lobe of the INM spectrum is consistent with the inherent structure approach to liquid dynamics.

We started by first establishing the relation between the localized nature of low-lying vibrational modes and their description with the soft-potential model. We assumed that defects are frozen in at the glass temperature, giving rise to stress in liquids. These properties define the model from which we then calculated the density of unstable modes. In the high- and low-temperature limit, we recovered exponen- tial frequency and temperature dependence that were previously found in computer simulations. We further found a lower (imaginary) frequency cutoff separating contributions from single- and double-well potentials to the unstable density of states. Using approximate formulas relating the fraction of unstable modes to the diffusion constant, we found Arrhenius temperature dependence of the diffusion constant with a crossover to Zwanzig-Bässler dependence for temperatures close to $T_{g}$. These two temperature regimes are known to correspond to landscapes with uniform and Gaussian barrier height distributions, respectively. The barrier height of a single mode is defined from the soft potential. We derived the above energy distributions in the appropriate limits. For a variety of systems (glass) transitions have been identified in experiments probing the short-time dynamics. Buchenau and Zorn have proposed a linear relation between the viscosity and the inverse of the mean-square displacement of the system's particles for high frequencies. We recovered this relation from our expression for the hightemperature limit of the diffusion constant, and thus identified the high-temperature limit with fast $\beta$-relaxation processes and the low-temperature limit with slow $\alpha$-relaxation processes.

Analytical theories for the entire INM spectrum that have previously been proposed are based on the connection between the Laplace transform of the return probability of a random walker and the phonon density of states in disordered systems [42]. Wu and Loring generalize this approach to higher dimensions, in which case atomic motions along $d$ orthogonal directions are coupled [43]. They use configuration-averaged transport properties of a random walker to calculate the frequency spectrum [44]. Using a two-body approximation to the self-energy, they derive analytical expressions for the density of states that are in good agreement with results from computer simulations of Lennard-Jones systems. The Green's-function approach to INM spectra is further elaborated on in a paper by Wan and Stratt [45]. Starting from expressions for the Green's function derived from the replica method, they use a diagrammatic formulation to incorporate many-body effects in their 
theory. This refined theory gives a better representation of the unstable lobe of the INM spectrum. In an earlier version of this theory [46], the distribution of the eigenvalues of the dynamic matrix follows the "semicircle law." Such a distribution is familiar from the theory of random matrices [47], and has recently been derived by Zwanzig for a highly connected random master equation [48]. The theories of Refs. [43] and [45] do not give simple analytic expressions for the frequency dependence of the stable and unstable lobe of the INM spectrum, so that a comparison with the present theory cannot readily be made. This would be highly desirable however, since the analytic expressions derived here have allowed us to associate unstable instantaneous normal modes with fast and slow dynamic processes in the liquid. The complexity of the Green's function formalism reflects the fact that the phononlike coordinates used in the random-walk approach do not easily describe modes localized in cooperative rearrangement regions of finite size [49].

In this paper, we considered the unstable density of states only for the limiting cases of low, $T \gtrsim T_{g}$, and high temperatures, $T \gg T_{g}$. For intermediate temperatures, MD simulations of a Lennard-Jones system indicate that the frequency dependence of the unstable density interpolates smoothly between these limiting cases. Indeed, we show [50] that an algebraic sum of the high- and low-temperature limits [cf. Eqs. (3.30) and (3.31)], gives an excellent fit to the unstable density for a broad temperature range $0.5<T<20$ (where $T$ is in reduced Lennard-Jones units). This form of the unstable density suggests that thermal stress varies in space, and the configuration space of the system consists of regions whose topographies are characterized by uniform and Gaussian distributions of barrier heights. It is shown in Ref. [51] that the number of particles in correlated regions can be estimated from an analysis of the eigenvectors of the dynamic matrix. Thus, instantaneous normal modes examine the energy landscape as well as spatial correlations in the liquid. Such spatial heterogeneity has recently been used to explain enhanced translational diffusion of large probe molecules in a supercooled liquid ( $o$-terphenyl) [52,53]. Spatial heterogeneity, in addition to dynamic heterogeneity, plays a major role in theories of the glass transition [54-57], and has been proposed as a possible mechanism for nonexponential relaxation in supercooled liquids [58].

\section{ACKNOWLEDGMENT}

This work has been supported by NSF Grant No. CHE9415216 (TK).
[1] J. C. Maxwell, Philos. Mag. 157, 49 (1867).

[2] J. Frenkel, Kinetic Theory of Liquids (Dover, New York, 1955).

[3] R. Zwanzig, J. Chem. Phys. 97, 4507 (1983).

[4] B. Madan, T. Keyes, and G. Seeley, J. Chem. Phys. 92, 7565 (1990); 94, 6762 (1991); G. Seeley, T. Keyes, and B. Madan, J. Chem. Phys. 95, 3847 (1991).

[5] R. M. Stratt, Acc. Chem. Res. 28, 201 (1995), and references therein.

[6] U. Mohanty, Adv. Chem. Phys. 89, 89 (1995).

[7] S. D. Bembenek and B. B. Laird, Phys. Rev. Lett. 74, 936 (1995); J. Chem. Phys. 104, 5199 (1996).

[8] B. B. Laird and H. R. Schober, Phys. Rev. Lett. 66, 636 (1991); H. R. Schober and B. B. Laird, Phys. Rev. B 44, 6746 (1991).

[9] T. Keyes, J. Chem. Phys. 101, 5081 (1992).

[10] G. V. Vijayadamodar and A. Nitzan, J. Chem. Phys. 103, 2169 (1995).

[11] P. Hänggi, P. Talkner, and M. Borkovec, Rev. Mod. Phys. 62, 251 (1990).

[12] R. Zwanzig, Proc. Natl. Acad. Sci. USA 85, 2029 (1988); H. Bässler, Phys. Rev. Lett. 58, 767 (1987).

[13] C. A. Angell, Science 267, 1924 (1995).

[14] For a review see, e.g., W. Götze, in Liquids, Freezing, and the Glass Transition, edited by D. Levesque, J. P. Hansen, and J. Zinn-Justin (Elsevier, New York, 1991).

[15] F. H. Stillinger and T. A. Weber, Science 225, 983 (1984).

[16] F. A. Lindemann, Z. Phys. 11, 609 (1910).

[17] T. R. Kirkpatrick and P. G. Wolynes, Phys. Rev. A 35, 3072 (1987)

[18] W. A. Phillips, J. Low Temp. Phys. 7, 351 (1972).

[19] P. W. Anderson, B. I. Halperin, and C. M. Varma, Philos. Mag. 25, 1 (1972).
[20] R. M. J. Cotterill and J. U. Madsen, Phys. Rev. B 33, 262 (1986).

[21] A. Heuer and R. J. Silbey, Phys. Rev. Lett. 70, 3911 (1993); Phys. Rev. B 48, 9411 (1993).

[22] U. Buchenau, H. M. Zhou, N. Nücker, K. S. Gilroy, and W. A. Phillips, Phys. Rev. Lett. 60, 1318 (1988).

[23] U. Buchenau, Yu. M. Galperin, V. L. Gurevich, and H. R. Schober, Phys. Rev. B 43, 5039 (1991).

[24] M. Goldstein, J. Chem Phys. 51, 3728 (1969).

[25] G. P. Johari and M. Goldstein, J. Chem. Phys. 53, 2372 (1970).

[26] U. Zürcher, T. Keyes, and B. B. Laird (unpublished).

[27] C. C. Yu and A. J. Leggett, Comments Condens. Matter Phys. 14, 231 (1988).

[28] V. G. Karpov and D. A. Parshin, Pis'ma Zh. Éksp. Teor. Fiz. 38, 536 (1983) [JETP Lett. 38, 648 (1983)].

[29] V. G. Karpov, M. I. Klinger, and F. N. Ignatiev, Zh. Éksp. Teor. Fiz. 84, 760 (1983) [Sov. Phys. JETP 65, 1641 (1983)].

[30] Yu. M. Galperin, V. L. Gurevich, and D. A. Parshin, Phys. Rev. B 32, 6873 (1985).

[31] A. P. Sokolov, Science 273, 1675 (1996).

[32] U. Buchenau, J. Mol. Struct. 296, 275 (1993).

[33] L. Gil, M. A. Ramos, A. Bringer, and U. Buchenau, Phys. Rev. Lett. 70, 182 (1993).

[34] The spectral dimensionality $\delta$ is defined $g(\omega) \sim \omega^{\delta-1}$. Using the scaling relation of E. Abrahams et al. [Phys. Rev. Lett. 42, 673 (1979)], R. Rammal and G. Toulouse have shown [J. Phys. (Paris), Lett. 44, L13 (1983)] that the conductance scales with length for fractals as $\zeta(L) \sim L^{\beta}$. Here, the exponent depends on the spectral dimensionality and the density dimensionality $\delta^{\prime}$ (the so-called Hausdorff dimensionality) $\beta=\delta^{\prime}[1-2 / \delta]$. That is, $\beta \leqslant 0$ for $\delta \leqslant 2$ so that $\delta=2$ is consistent with localized behavior. 
[35] L. Ferrari, W. A. Phillips, and G. Russo, Europhys. Lett. 3, 611 (1987).

[36] S.-P. Chen, T. Egami, and V. Vitek, Phys. Rev. B 37, 2440 (1988).

[37] U. Buchenau, Philos. Mag. 65, 303 (1992).

[38] T. Keyes, J. Chem. Phys. 103, 9810 (1995).

[39] F. H. Stillinger, Science 267, 1935 (1995).

[40] U. Buchenau and R. Zorn, Europhys. Lett. 18, 523 (1992).

[41] D. Thirumalai, R. D. Mountain, and T. R. Kirkpatrick, Phys. Rev. A 39, 3563 (1989).

[42] S. Alexander, J. Bernasconi, W. R. Schneider, and R. Orbach, Rev. Mod. Phys. 53, 175 (1981).

[43] T.-M. Wu and R. F. Loring, J. Chem. Phys. 97, 8568 (1992).

[44] C. R. Gochanour, H. C. Andersen, and M. D. Fayer, J. Chem. Phys. 70, 4254 (1979).

[45] Y. Wan and R. M. Stratt, J. Chem. Phys. 100, 5123 (1994).

[46] B.-C. Xu and R. M. Stratt, J. Chem. Phys. 92, 1923 (1990).

[47] M. L. Mehta, Random Matrices and the Statistical Theory of Energy Levels (Academic, London, 1967).

[48] R. Zwanzig, J. Chem. Phys. 103, 9397 (1995).

[49] G. Adam and J. H. Gibbs, J. Chem. Phys. 43, 139 (1965).
[50] U. Zürcher, T. Keyes, and G. V. Vijayadamodar (unpublished).

[51] R. O. Rosenberg, D. Thirumalai, and R. D. Mountain, J. Phys. Condens. Matter 1, 2109 (1989).

[52] F. Fujara, B. Geil, H. Sillescu, and G. Fleischer, Z. Phys. B 88, 195 (1992); I. Chang, F. Fujara, B. Geil, G. Heuberger, T. Mangel, and H. Sillescu, J. Non-Cryst. Solids 172-174, 248 (1994).

[53] M. T. Cicerone and M. D. Ediger, J. Chem. Phys. 103, 5684 (1995); 104, 7210 (1996).

[54] G. S. Grest and M. H. Cohen, Adv. Chem. Phys. 48, 455 (1981).

[55] T. R. Kirkwood, D. Thirumalai, and P. G. Wolynes, Phys. Rev. A 40, 1045 (1989).

[56] F. H. Stillinger and J. A. Hodgdon, Phys. Rev. E 50, 2064 (1994).

[57] D. Kivelson, S. A. Kivelson, X. Zhao, Z. Nussinov, and G. Tarjus, Physica A 219, 27 (1995).

[58] J. T. Bendler and M. F. Shlesinger, J. Phys. Chem. 96, 3970 (1992). 